\title{
High fetal bisphenol A exposure enhances IL22 secretion: A proinflammatory cytokine
}

\author{
Ozel Yuruker, ${ }^{1}$ Ceyhun Dalkan, ${ }^{2}$ Murat Uncu, ${ }^{3}$ Osman Yetkin, ${ }^{3}$ Arzu Babayigit, ${ }^{4}$ Nerin N. Bahceciler Onder ${ }^{2}$
}

\begin{abstract}
Background: Bisphenol A (BPA) is an industrial product, widely used in human consumed types of equipment that can be transmitted orally, by inhalation or through dermal absorption and is detectable in many body fluids including cord blood. A correlation between BPA concentration in maternal serum and cord blood has been demonstrated previously, suggesting a possible transfer of BPA via the transplacental path.
\end{abstract}

Objective: Our objective is to determine the impact of cord blood BPA level on cytokine responses.

Methods: In this cross-sectional study, healthy pregnant women who delivered healthy newborns followed by the Obstetrics and Gynecology Department between September 2016 to June 2017 were enrolled. Cord blood samples were obtained and BPA and IL4, IL5, IL10, IL17, IL22, IFN gama and TGF beta levels were studied by ELISA.

Results: Among 197 deliveries, 176 of them were included in the study. Due to lack of cut-off value, BPA levels were stratified as percentiles. No statistically significant difference was detected in comparison of cytokine levels based on BPA concentrations below and above the $25^{\text {th }}$ and $50^{\text {th }}$ percentiles. Significantly higher IL22 levels $(p=0.007)$ and increased ratio of IL22/TGF $\beta(p=0.04)$ were detected in those with BPA level above $75^{\text {th }}$ percentile $(>19.16 \mathrm{ng} / \mathrm{ml})$ compared to the below group.

Conclusions: This in vivo real-life study demonstrated that very high BPA levels in cord blood of expectant mothers enhances IL22 secretion in cord blood which is a proinflammatory cytokine. Studies evaluating long term immunological effects on those highly exposed newborns are necessitated.

Key words: Cord blood, cytokines, Interleukin 22, maternal exposure, newborn

\section{From:}

${ }^{1}$ Department of Immunology, Faculty of Medicine,

University of Kyrenia, Kyrenia, Cyprus

${ }^{2}$ Department of Pediatrics, Faculty of Medicine, Near East University,

Nicosia, Cyprus

${ }^{3}$ Department of Biochemistry, Faculty of Medicine,

Near East University, Nicosia, Cyprus

${ }^{4}$ Department of Pediatrics, Faculty of Medicine, University of Kyrenia, Kyrenia, Cyprus

\section{Introduction}

Bisphenol A (BPA) is widely used in the manufacturing of many human consumer products such as beverage/food cans, plastic bottles, cosmetics, toys and medical types of equipment, etc. ${ }^{1,2}$

BPA is readily passed into those products, when exposed to high temperatures, and can be transmitted to human orally, by inhalation or through dermal absorption. ${ }^{3}$

\section{Corresponding author:}

Ozel Yuruker

Department of Immunology, Faculty of Medicine,

University of Kyrenia, Kyrenia, Cyprus

E-mail: ozel.yuruker@yahoo.com,ozel.yuruker@kyrenia.edu.tr

Bio-monitoring studies have reported that BPA is detectable in many body fluids such as blood serum, urine, amnionic fluid, breast milk, and cord blood. ${ }^{4}$ Previous studies demonstrated that there is a correlation between BPA concentration in maternal serum and cord blood, which raises the possibility of the transfer of BPA via the transplacental path. ${ }^{5,6}$ 
BPA is accepted as an endocrine-disrupting chemical (EDC) in humans, and the accumulation of the compound has a significant impact on the endocrine system..$^{3,7,8,9}$ Besides causing an endocrine system disruption, fetal BPA exposure was associated with various autoimmune and inflammatory diseases, including allergy and asthma later in life. ${ }^{1,6,10}$ Studies have shown that the immune system is sensitive to BPA exposure, and the fetal time is a critical period for the development of the immune system. This susceptibility may result from the immaturity of fetal organs and undeveloped detoxification systems. ${ }^{11}$

Previously, it has been hypothesized that BPA may have immune dysregulating effects such as susceptibilty to allergic immune responses. ${ }^{10,12}$ Several studies on experimental animal models have shown that the effect of prenatal BPA exposure may have a modifying impact on the synthesis of cytokines. ${ }^{1,9,12-14}$ Studies are indicating that BPA exposure may be linked to immunological outcomes in humans, however, as the human studies have limitations, the link between BPA exposure during pregnancy and immune effects in humans remains uncertain. ${ }^{15}$

Our objective in this study was to determine the impact of different cord blood BPA levels on cytokine responses including IFN- $\gamma$, TGF- $\beta$, IL10, IL4, IL5, IL17A, IL22. The shifts in the balance of those cytokines may have impact on the skewing of innate and specific immune responses. Based on those preliminary results, hereby, we aimed to evaluate whether the transplacental BPA exposure has an impact on the cord blood cytokine milieu.

\section{Materials and methods}

\section{Study Population and Anthropometric Data}

The Institutional Ethics Committee approval was obtained before the study (YDU/2015/32-215), and informed parental consent was obtained for each participant.

In this cross-sectional study, All expentant mothers with a healthy pregnancy process who had no known chronic diseases and chronic drug addiction followed by the Obstetrics and Gynecology Department of the University hospital between September 2016 and June 2017 were invited to participate in the study. Pregnant mothers who accepted to participate and delivered healthy newborns were included in the study. Exclusion criteria were: Delivery of newborns with congenital anomalies, perinatal asphyxia and major surgical operation during labour. Cord blood samples were collected during labour for BPA and cytokine measurements.

\section{Blood Sampling and BPA Level Measurement}

Cord blood samples from the umbilical vein were obtained at birth and collected into BPA-free polystyrene tubes (BD Diagnostics Preanalytical Systems, BE). Each blood sample was left to coagulate for 30 minutes. Then samples were centrifuged at $2000 \mathrm{~g}$ for 10 minutes at room temperature to obtain serum, which was stored in aliquots in BPA-free Eppendorf (Eppendorf $\mathrm{AG}, \mathrm{GE}$ ) vials at $-80^{\circ} \mathrm{C}$ until analysis. On the day of analysis, the aliquots were brought to room temperature and thoroughly vortexed before the analysis.
Biological markers were analyzed by sandwich enzymelinked immunosorbent assays (ELISAs) kits for BPA (General Bisphenol A ELISA, MyBioSource, Inc., San Diego, California, USA), TGF- $\beta$ (ELISA, e-bioscience, Vienna, Austria), IL10 (DIASource Immunoassays SA, Louvain-LA-Neuve, Belgium), IL4 (ELISA, e-bioscience, Vienna, Austria), IL5 (ELISA, e-bioscience, Vienna, Austria), IL17a (ELISA, e-bioscience, Vienna, Austria), IL22 (ELISA, e-bioscience, Vienna, Austria), IFN- $\alpha$ (ELISA, e-bioscience, Vienna, Austria). The methods of measurements were carried out according to the manufacturer's instructions.

ELISAs were read with a Spectramax M5 Series Multi-Mode Microplate Reader (Molecular Devices, Sunnyvale, California, USA). The standard curves were created with a software program called Softmax Pro 5.2 that is cable of generating four parametric logistics (4PL) curve-fit.

\section{Statistical Analysis}

Statistical analysis was performed using SPSS version 22 for Macintosh (SPSS Inc., Chicago, Illinois, USA). The results were expressed as mean and standard deviation of the mean. Cord blood cytokines were compared with below and above the $25^{\text {th }}, 50^{\text {th }}$, and $75^{\text {th }}$ percentile of cord blood BPA levels. To determine the relationship between principal variables and the other continuous variables, the Pearson correlation test was used. The Mann-Whitney $U$ test was used to determine the relationship between grouped variables. A p-value of less than 0.05 was considered statistically significant.

\section{Results}

Among 197 deliveries, 21 of them were excluded based on inclusion and exclusion criteria, leaving 176 healthy term labors in this study. Seventy-seven $(43.7 \%)$ of newborns were male, and 99 (56.3\%) were female. The mean gestational week was $38.20 \pm 1.8$ weeks. Demographic characteristics of delivery and newborns are presented in Table 1. Mean values of the cord blood BPA and cytokine measurements are shown in Table 2.

\section{Table 1. Demographic characteristics of study group}

$\begin{array}{lc}\text { Characteristics (n:176) } & \\ \text { Male (n/\%) } & 77 / 43.70 \\ \text { Female (n/\%) } & 99 / 56.30 \\ \text { Caeserean section (n/\%) } & 167 / 94.80 \\ \text { Normal delivery (n/\%) } & 9 / 5.20 \\ \text { Birth weight (gr) (Mean } \pm \text { SD) } & 3156.76 \pm 493.45 \\ \text { Birth height (cm) (Mean } \pm \text { SD) } & 48.28 \pm 2.04 \\ \text { Head circumference (cm) (Mean } \pm \text { SD) } & 34.14 \pm 1.74\end{array}$


Table 2. Cord blood BPA and cytokine levels

\begin{tabular}{|lc|}
\hline Parameter & $\begin{array}{c}\text { Concentration }(\mathbf{n g} / \mathbf{m l}) \\
\text { Mean } \pm \text { SD }\end{array}$ \\
\hline BPA & $14.5 \pm 6.20$ \\
\hline IFN- $\gamma$ & $2.52 \pm 4.17$ \\
\hline TGF- $\beta$ & $40.54 \pm 22.70$ \\
\hline IL-10 & $81.18 \pm 256.62$ \\
\hline IL-4 & $8.85 \pm 10.30$ \\
\hline IL-5 & $1.86 \pm 4.96$ \\
\hline IL-17A & $0.96 \pm 0.81$ \\
\hline IL-22 & $2.19 \pm 10.65$ \\
\hline
\end{tabular}

For further comparisons based on the concentration of BPA levels, the measured BPA levels were stratified as percentiles of $25^{\text {th }}, 50^{\text {th }}, 75^{\text {th }}$ to evaluate the effect of different levels of exposure to BPA on cytokine synthesis. The $25^{\text {th }}, 50^{\text {th }}$, and $75^{\text {th }}$ percentiles of cord blood BPA levels of the whole group were found to be $9.84 \mathrm{ng} / \mathrm{ml}, 13.96 \mathrm{ng} / \mathrm{ml}, 19.16 \mathrm{ng} / \mathrm{ml}$, respectively (Figure 1).

Below versus above percentile comparisons were made for all cytokine measurements.

There was no statistically significant difference in cytokine levels in the comparisons of BPA concentrations below and above the $25^{\text {th }}$ and $50^{\text {th }}$ percentile groups (Table 3 ). Whereas, the comparison between BPA below versus above $75^{\text {th }}$ percentile groups revealed statistically significant higher IL22 levels $(p=0.007)$ in the more exposed group (above $75^{\text {th }}$ percentile group) (Table 3 ).

To evaluate whether there is an association between higher BPA level and ratio of cytokine levels in cord blood, multiple comparisons were performed for below versus above $25^{\text {th }}, 50^{\text {th }}, 75^{\text {th }}$ percentiles for the following ratios: IFN $\gamma / \mathrm{TGF} \beta$, IL4/TGF $\beta$, IL5/TGF $\beta$, IL17/TGF $\beta$, IL22/TGF $\beta$ and IFN $\gamma$ / IL10, IL4/IL10, IL5/IL10, IL17/IL10, IL22/IL10.

There was a statistically significant increase in IL22/TGF $\beta$ $(p=0.04)$ and a decrease in IL5/TGF $\beta(p=0.05)$ ratios in those with BPA level above $75^{\text {th }}$ percentile compared to the below group (Table 4). No statistically significant difference
25

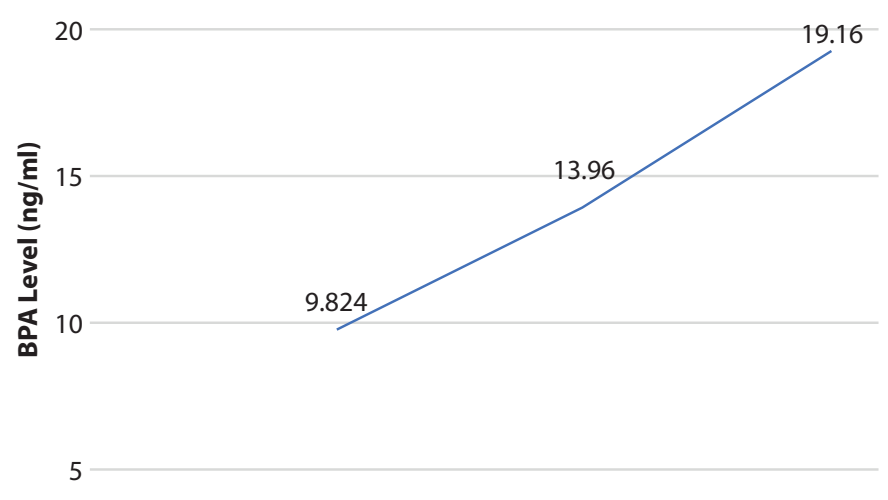

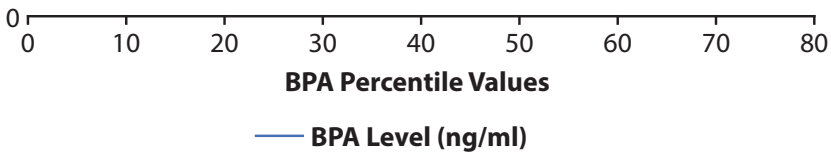

Figure 1. The measured BPA levels were subgrouped as percentiles. $25^{\text {th }}, 50^{\text {th }}, 75^{\text {th }}$ percentile values of measured cord blood BPA values were calculated $(9.84 \mathrm{ng} / \mathrm{ml}, 13.96 \mathrm{ng} / \mathrm{ml}$, $19.16 \mathrm{ng} / \mathrm{ml}$ respectively). Below versus above percentile comparisons were made for all cytokine measurements.

Table 4. Comparison of each cytokine level relative to TGF-beta level based on $\leq$ and $>75^{\text {th }}$ percentiles.

\begin{tabular}{lccc}
\multicolumn{1}{|c}{ Percentile $^{*}$} & $\begin{array}{c}\text { BPA } \leq 75^{\text {th }} \\
\text { Mean } \pm \text { SD }\end{array}$ & $\begin{array}{c}\text { BPA }>75^{\text {th }} \\
\text { Mean } \pm \text { SD }\end{array}$ & P Value \\
\hline IFN $\gamma /$ TGF $\beta$ & $0.15 \pm 0.40$ & $0.09 \pm 0.16$ & 0.12 \\
IL4/TGF $\beta$ & $0.58 \pm 1.50$ & $0.29 \pm 0.59$ & 0.09 \\
IL5/TGF $\beta$ & $0.13 \pm 0.59$ & $0.03 \pm 0.07$ & 0.05 \\
IL17A/TGF $\beta$ & $0.07 \pm 0.16$ & $0.04 \pm 0.07$ & 0.12 \\
IL22/TGF $\beta$ & $0.01 \pm 0.10$ & $0.16 \pm 0.88$ & 0.04 \\
\hline
\end{tabular}

${ }^{*} 75^{\text {th }}$ percentile BPA value is $19.16 \mathrm{ng} / \mathrm{ml}$

Table 3. Comparison of cord blood cytokine levels according to $25^{\text {th }}, 50^{\text {th }}$, and $75^{\text {th }}$ percentiles

\begin{tabular}{|c|c|c|c|c|c|c|c|c|c|}
\hline $\begin{array}{l}\text { Cytokine levels } \\
(\mathrm{ng} / \mathrm{ml})\end{array}$ & $\begin{array}{c}\leq 25^{\text {th }} \\
\text { Mean } \pm \text { SD }\end{array}$ & $\begin{array}{c}>25^{\text {th }} \\
\text { Mean } \pm \text { SD }\end{array}$ & $P$ & $\begin{array}{c}\leq 50^{\text {th }} \\
\text { Mean } \pm \text { SD }\end{array}$ & $\begin{array}{c}>50^{\text {th }} \\
\text { Mean } \pm \text { SD }\end{array}$ & $\mathbf{P}$ & $\begin{array}{c}\leq 75^{\text {th }} \\
\text { Mean } \pm \text { SD }\end{array}$ & $\begin{array}{c}>75^{\text {th }} \\
\text { Mean } \pm \text { SD }\end{array}$ & $P$ \\
\hline IFN- $\gamma$ & $2.59 \pm 4.50$ & $2.3 \pm 2.97$ & 0.7 & $2.05 \pm 2.51$ & $2.99 \pm 5.30$ & 0.1 & $2.4 \pm 3.70$ & $2.8 \pm 5.30$ & 0.7 \\
\hline TGF- $\beta$ & $40.19 \pm 52.60$ & $41.58 \pm 23.36$ & 0.7 & $42.9 \pm 23.10$ & $38.19 \pm 22.20$ & 0.2 & $41.5 \pm 23.50$ & $37.7 \pm 10.20$ & 0.3 \\
\hline IL-10 & $86.2 \pm 289.00$ & $85.96 \pm 115.90$ & 0.5 & $57.3 \pm 90.50$ & $105.3 \pm 350$ & 0.2 & $61.6 \pm 113.90$ & $139.99 \pm 473.00$ & 0.3 \\
\hline IL-4 & $8.9 \pm 10.50$ & $8.62 \pm 9.50$ & 0.9 & $8.7 \pm 11.20$ & $9.04 \pm 9.40$ & 0.8 & $9.2 \pm 11.40$ & $7.6 \pm 6.10$ & 0.2 \\
\hline IL-5 & $1.85 \pm 4.70$ & $1.89 \pm 5.60$ & 0.9 & $1.78 \pm 0.92$ & $5.1 \pm 1.94$ & 0.8 & $2.01 \pm 5.20$ & $1.2 \pm 3.90$ & 0.3 \\
\hline IL-17A & $0.97 \pm 0.80$ & $0.95 \pm 0.81$ & 0.9 & $0.87 \pm 0.80$ & $1.05 \pm 0.81$ & 0.1 & $0.97 \pm 0.80$ & $0.96 \pm 0.80$ & 0.9 \\
\hline IL-22 & $2.03 \pm 10.20$ & $2.5 \pm 12.03$ & 0.8 & $2.5 \pm 10.10$ & $1.8 \pm 10.00$ & 0.7 & $0.1 \pm 2.10$ & $2.91 \pm 12.20$ & 0.007 \\
\hline
\end{tabular}

${ }^{*} 25^{\text {th }}, 50^{\text {th }}, 75^{\text {th }}$ percentile values are $9.82 \mathrm{ng} / \mathrm{ml}(\mathrm{n}=44), 13.96 \mathrm{ng} / \mathrm{ml}(\mathrm{n}=45), 19.16 \mathrm{ng} / \mathrm{ml}(\mathrm{n}=87)$ respectively 
was detected in comparisons of the ratio to IL10 of the studied cytokines (data not shown).

\section{Discussion}

Recent studies have revealed that the potential role of endocrine-disrupting chemicals such as BPA may also have an impact on the modification of cytokine responses. ${ }^{15}$ Previously conducted in vitro human studies, as well as, murine studies revealed that BPA could pass through the placental barrier, and there is a correlation between maternal and placental BPA levels. ${ }^{16}$ Moreover, the correlation between maternal and cord blood BPA concentrations has been demonstrated previously. ${ }^{5,6}$ Therefore, in this study, we considered to measure only cord blood BPA levels based on those previous results.

BPA concentrations have been adjusted to supraphysiologic levels in in-vitro and murine studies for the evaluation of their effect on the immune responses. However, those studies do not represent real-life exposures. Hereby, we aimed to measure the impact of real-life BPA exposure during pregnancy by means of measuring cord blood BPA and evaluate its impact on cytokine responses in cord blood.

Cytokines are substances that enable the activity, stimulation, and intra-communication of immune cells, which in turn affects the development of the innate and $\mathrm{T}$ cell immune responses. Several murine studies have demonstrated that environmental in utero costimulatory signals have an impact on $\mathrm{T}$ helper cell differentiation at the early fetal immune system development. ${ }^{17}$ Limited studies are showing the effect of prenatal BPA exposure on the differentiation of newborn $\mathrm{T}$ helper cells. Meanwhile, ILCs represent innate immunity which mediate both pro-inflammatory and anti-inflammatory responses not requiring recombination or expansion from memory cells, but responding to antigens rapidly through cytokine secretion. These cells are classified as 3 subgroups ILC1, ILC2, ILC3- based on their secreted cytokines and transcription factor expressed. ILCs are programmed at the embryogenic stage suggesting their role in the neonatal innate immune system with a higher number and activity level during the neonatal period, unlike the other innate immune cells including neutrophils and monocytes. ${ }^{17}$

In previous studies, it has been demonstrated that there is a dose-related impact of BPA on fetal growth, adiposity, and neonatal complications. There were no significant differences in birth weight, length, and head circumference based on different cord BPA levels. ${ }^{2}$ To our knowledge, this is the first in-vivo study in humans that investigated the cord blood BPA exposure level on the cytokine secretion in cord blood. Cord blood BPA was accepted as representative of maternal exposure based on results of previous studies., ${ }^{5,6}$ Therefore, we aimed to show the association between the cord blood BPA and cytokine levels including IFN- $\gamma$, TGF- $\beta$, IL10, IL4, IL5, IL17A, and IL22. This question was prompted by the fact that placental exposure may alter immune system development and may promote the risk of development of certain immune system disorders in later life. ${ }^{10}$
In the current study, cord blood BPA measurements were divided into percentiles $25^{\text {th }}, 50^{\text {th }}$, and $75^{\text {th }}$ to detect the doseresponse effect on the cytokine secretion. IFN- $\gamma$ is produced by a variety of immune cells including ILC1 and Th1 cells playing an essential role in the inflammatory reactions and viral infections. TGF- $\beta$ and IL10 are anti-inflammatory cytokines that have regulatory functions, aswell. IL4 and IL 5 play an important role in the development of certain immune disorders, particularly allergies. IL17A and IL22 are proinflammatory cytokines that are produced mainly by activated ILC3 and Th17 cells. ${ }^{18}$ The proinflammatory activity of Th17 cells can be beneficial to the host during infection. However, uncontrolled Th17 activation has been linked to several autoimmune and autoinflammatory pathologies such as multiple sclerosis, arthritis, and lupus. ${ }^{19}$

The in vitro experiments using human cord blood cells have shown that neonates have a shallow frequency or complete absence of Th17 cells due to the lower expression of RORC mRNA. ${ }^{20}$ Meanwhile, several experimental murine studies evaluated the impact of various concentrations of BPA exposure on the differentiation of $\mathrm{T}$ helper cells into functional subpopulations of Th1, Th2, Th17, and Treg. The preferential development into those subgroups varied between studies, and results were conflicting. ${ }^{15}$ It has been reported that gestational exposure of mice to BPA results in increased numbers of Th17 cells and IL17 mRNA expression. ${ }^{1}$ BPA induced enhanced Th17 cell activity could provide a mechanism through which BPA might induce inflammatory responses. In a recent murine study prenatal BPA exposure of mothers resulted in intestinal and systemic increased release of IL17 and Th17 - Th1 responses besides decreased Treg responses in the offsprings. In addition the offsprings demonstrated a decreased frequency of IL22 producing ILC3 cells in the small intestinal lamina propria. ${ }^{21}$

Currently, the immunological effect of BPA on human immune responses is unclear. In the current study, cord blood IL22 levels, and the ratio of IL22/TGF $\beta$ with high BPA exposure $\left(>75^{\text {th }}\right.$ percentile) were significantly higher. IL22 is a member of IL10 superfamily, which is the class of potent mediators of inflammatory responses. High IL22 levels generally correlate with the increased ILC 3 and Th17 cell activity. ILC3 population is enriched at the mucosal sites during lynphoid tissue organegenesis in fetal life and regulates intestinal hemostasis in the long term through the secretion of IL22. ${ }^{22}$

Also, in the current study ratio of IL5 which is secreted mainly from ILC2 and Th2 cells to the regulatory cytokine TGF $\beta 17$ was demonstrated to be low with borderline significance $(p=0.05)$ in those with higher exposure to BPA $\left(>75^{\text {th }}\right.$ percentile). The role of BPA exposure in the development of allergy and allergic diseases is conflicting. ${ }^{10,12,15,23-25}$

The major limitation of the current study is that only cord blood cytokine measurements were performed which does not reveal the source of IL22 secretion. Determination of the source cell, such as T helper or ILC, of any cytokine needs further testing by flow cytometric analysis. Another limitation is the absence of long-term neonatal follow-up monitorization of the serum cytokine levels. 
In conclusion, the results of this in vivo real-life study demonstrated that very high levels of cord blood (more than $75^{\text {th }}$ percentile) BPA might enhance the proinflammatory responses through the induction IL22 release. The impact of neonatal cytokine shifts on the development of neonatal immune responses may lead to immune system disorders such as allergies and autoimmune diseases later in life. Those patients should be followed up in the long run, and possible adverse effects of BPA exposure should be further investigated.

\section{Acknowledgements}

The authors report no conflict of interest

This study was supported by Near East University [DESAM-134]

\section{References}

1. Luo S, Li Y, Li Y, Zhu Q, Jiang J, Wu C, et al. Gestational and lactational exposure to low-dose bisphenol A increases Th17 cells in mice offspring. Environ Toxicol Pharmacol. 2016;47:149-58.

2. Dalkan C, Uncu M, Duran S, Bahceciler NN. Association of cord blood bisphenol A (BPA) with cord blood adiponectin, leptin, fetal growth, adiposity and neonatal complications in a newborn cohort. J Matern Fetal Neonatal Med. 2019:1-6.

3. Sanlidag B, Dalkan C, Yetkin O, Bahceciler NN. Evaluation of Dose Dependent Maternal Exposure to Bisphenol A on Thyroid Functions in Newborns. J Clin Med. 2018;7(6)

4. Rochester JR. Bisphenol A and human health: a review of the literature. Reprod Toxicol. 2013;42:132-55.

5. Mammadov E, Uncu M, Dalkan C. High Prenatal Exposure to Bisphenol A Reduces Anogenital Distance in Healthy Male Newborns J Clin Res Pediatr Endocrinol. 2018;10(1):25-9.

6. Liao SL, Tsai MH, Lai SH, Yao TC, Hua MC, Yeh KW, et al. Prenatal exposure to bisphenol-A is associated with Toll-like receptor-induced cytokine suppression in neonates. Pediatr Res. 2016;79(3):438-44.

7. Chevrier J, Gunier RB, Bradman A, Holland NT, Calafat AM, Eskenazi $B$, et al. Maternal urinary bisphenol a during pregnancy and maternal and neonatal thyroid function in the CHAMACOS study. Environ Health Perspect. 2013;121(1):138-44.

8. Giesbrecht GF, Ejaredar M, Liu J, Thomas J, Letourneau N, Campbell $\mathrm{T}$, et al. Prenatal bisphenol an exposure and dysregulation of infant hypothalamic-pituitary-adrenal axis function: findings from the APrON cohort study. Environ Health. 2017;16(1):47.

9. Rogers JA, Metz L, Yong VW. Review: Endocrine disrupting chemicals and immune responses: a focus on bisphenol-A and its potential mechanisms. Mol Immunol. 2013;53(4):421-30.
10. Ashley-Martin J, Dodds L, Levy AR, Platt RW, Marshall JS, Arbuckle TE. Prenatal exposure to phthalates, bisphenol A and perfluoroalkyl substances and cord blood levels of IgE, TSLP and IL-33. Environ Res. 2015;140:360-8.

11. Barr DB, Bishop A, Needham LL. Concentrations of xenobiotic chemicals in the maternal-fetal unit. Reprod Toxicol. 2007;23(3):260-6.

12. Midoro-Horiuti T, Tiwari R, Watson CS, Goldblum RM. Maternal bisphenol a exposure promotes the development of experimental asthma in mouse pups. Environ Health Perspect. 2010;118(2):273-7.

13. Ohshima Y, Yamada A, Tokuriki S, Yasutomi M, Omata N, Mayumi M. Transmaternal exposure to bisphenol a modulates the development of oral tolerance. Pediatr Res. 2007;62(1):60-4.

14. Lee J, Lee SJ, Lim KT. CTB glycoprotein $(75 \mathrm{kDa})$ inhibits IgE releasing, TNF-alpha and IL-6 expressed by bisphenol A in vivo and in vitro. Food Chem Toxicol. 2012;50(6):2109-17.

15. Kimber I. Bisphenol A and immunotoxic potential: A commentary. Regul Toxicol Pharmacol. 2017;90:358-63.

16. Pergialiotis V, Kotrogianni P, Christopoulos-Timogiannakis E, Koutaki D, Daskalakis G, Papantoniou N. Bisphenol A and adverse pregnancy outcomes: a systematic review of the literature. J Matern Fetal Neonatal Med. 2018;31(24):3320-7.

17. Yu JC, Khodadadi H, Malik A et al. Innate Immunity of Neonates and Infants. Frontiers in immunology 91759 (2018).

18. Gagliani N, Huber S. Basic Aspects of T Helper Cell Differentiation. Methods Mol Biol. 2017;1514:19-30.

19. Waite JC, Skokos D. Th17 response and inflammatory autoimmune diseases. Int J Inflam. 2012;2012:819467.

20. de Roock S, Stoppelenburg AJ, Scholman R, Hoeks S, Meerding J, Prakken BJ, et al. Defective TH17 development in human neonatal T cells involves reduced RORC2 mRNA content. J Allergy Clin Immunol. 2013;132(3): 754-6 e3.

21. Malaise Y, Menard S, Cartier C et al. Consequences of bisphenol a perinatal exposure on immune responses and gut barrier function in mice. Arch Toxicol 92(1), 347-358 (2018).

22. Van De Pavert SA, Vivier E. Differentiation and function of group 3 innate lymphoid cells, from embryo to adult. International immunology 28(1), 35-42 (2016)

23. Nakajima Y, Goldblum RM, Midoro-Horiuti T. Fetal exposure to bisphenol A as a risk factor for the development of childhood asthma: an animal model study. Environ Health. 2012;11:8.

24. Spanier AJ, Kahn RS, Kunselman AR, Hornung R, Xu Y, Calafat AM, et al. Prenatal exposure to bisphenol A and child wheeze from birth to 3 years of age. Environ Health Perspect. 2012;120(6):916-20.

25. Donohue KM, Miller RL, Perzanowski MS, Just AC, Hoepner LA, Arunajadai S, et al. Prenatal and postnatal bisphenol A exposure and asthma development among inner-city children. J Allergy Clin Immunol. 2013;131(3):736-42. 